\section{OC-128 VARIATIONS IN THE GENE ENCODING C REACTIVE PROTEIN SUGGEST THAT CRP IS A CANDIDATE SUSCEPTIBILITY GENE FOR INFLAMMATORY BOWEL DISEASE IN THE SCOTTISH PAEDIATRIC POPULATION}

doi:10.1136/gut.2011.239301.128

P Henderson, ${ }^{1 *} \mathrm{~J}$ van Limbergen, ${ }^{2} \mathrm{~N}$ H Anderson, ${ }^{3} \mathrm{~F} \mathrm{~L}$ Cameron, ${ }^{4} \mathrm{E}$ Cameron, ${ }^{1} \mathrm{R} \mathrm{K}$ Russell, ${ }^{4} \mathrm{~J}$ Satsangi, ${ }^{5}$ D C Wilson ${ }^{1}$ Child Life and Health, University of Edinburgh, Edinburgh, UK; ${ }^{2}$ Department of Paediatric Gastroenterology, Hepatology and Nutrition, University of Toronto, Toronto, Canada; ${ }^{3}$ Centre for Population Health Sciences, University of Edinburgh, Edinburgh, UK; ${ }^{4}$ Department of Paediatric Gastroenterology, Royal Hospital for Sick Children, Glasgow, UK ${ }^{5}$ Gastrointestinal Unit, University of Edinburgh, Edinburgh, UK

Introduction $\mathrm{C}$ reactive protein (CRP) is an acute phase reactant which may possibly link innate and adaptive immunity by binding proteins such as complement factor H. IBD patients with an elevated serum CRP have demonstrated a superior response to biological therapies suggesting that CRP may also play a role in IBD pathogenesis. In addition, a proportion of IBD patients do not have a raised CRP even in the presence of severe inflammatory disease. We aimed to assess the genewide association signal of the CRP gene to IBD and Crohn's disease (CD) susceptibility by means of a detailed haplotypetagging investigation.

Methods 1215 subjects consisting of 465 robustly phenotyped patients diagnosed with inflammatory bowel disease (IBD) $<17$ years within Scotland (311 CD, 111 UC and 43 IBD-U) and 797 parents (284 complete trios) were genotyped for seven single nucleotide polymorphisms (SNPs). 5 CRP-tagging SNPs were selected using HapMap data (using solid spine of LD, MAF > 0.1): rs1417938, rs1205, rs1130864, rs1935193 and rs11265263. Two additional SNPs, previously shown to influence serum CRP levels (rs3091244 and rs1800947) were also genotyped. Detailed phenotypic characteristics of this cohort were previously described. Detailed single marker and haplotype analysis by transmission disequilibrium testing (ParenTDT) was carried out using Haploview (with permutation analysis, $\mathrm{n}=100$ 000).

Results After stringent permutation analysis the rs1417938 A allele, located in the first 5' intron of the CRP gene, showed significant over transmission to affected IBD patients $(\mathrm{p}=0.0446)$. The 7-marker haplotype ACACATC also showed significant distortion of transmission $(p=0.0068)$ This same 7-marker haplotype was also significantly associated with Crohn's disease $(p=0.0165)$. Using a sliding-haplotype analysis to assess the extent of the IBD and CD-associated signal from the 5' end to the 3'UTR of the CRP coding sequence demonstrated that the strongest signal ( $p=0.0013$ for IBD, $p=0.0050$ for CD) was achieved with a haplotype spanning the entire CRP gene.

Conclusion We have demonstrated that in a well defined population, using analysis that eliminates the need for population stratification, that inherited variation of the haplotype containing CRP is associated with IBD and CD susceptibility. Our analysis indicates that it is now warranted to submit the $C R P$ gene to further deep sequencing and to re-evaluate the contribution of CRP to IBD pathogenesis beyond its usefulness as an inflammatory marker.

Competing interests None.

Keywords genetics, inflammatory bowel disease, paediatrics, susceptibility. 